\title{
Improving energy efficiency in optical cloud networks by exploiting anycast routing
}

\author{
Jens Buysse* $^{* \mathrm{a}}$, Cicek Cavdar ${ }^{\mathrm{b}}$, Marc De Leenheer $^{\mathrm{a}}$, Bart Dhoedt ${ }^{\mathrm{a}}$, Chris Develder $^{\mathrm{a}}$ \\ ${ }^{a}$ Ghent University - INTEC-IBCN, G. Grommenlaan 8 bus 201, BE-9050 Gent, Belgium \\ ${ }^{\mathrm{b}}$ Royal Institute of Technology (KTH), ICT, Isafjordsgata 22, $16440 \mathrm{Kista}$, Sweden
}

\begin{abstract}
Exploiting anycast routing significantly reduces optical network and server energy usage. In this work we present a case study showing that intelligently selecting destinations and routes thereto, while switching off unused (network) elements, cuts power consumption by around $20 \%$ and saves network resources by $29 \%$.
\end{abstract}

Keywords: Energy efficiency, Anycast routing, Unicast routing, Integer Linear Programming, Optical Networks

\section{INTRODUCTION}

Motivated by the fact that ICT accounts for 2-10\% of worldwide power consumption and predictions that this will continue to increase [1], recently many works have investigated possible power saving mechanisms. In this paper, we focus on optical network infrastructure, which globally represents around $0.1 \mathrm{GW}$ of power, mainly stemming from routers. The scenario studied in this paper mainly addresses optical backbone networks [2]. Specifically, we study reduction of energy consumption in the context of clouds supported by an optical network, by proposing Energy Efficient (EE) routing which minimizes energy consumption of the optical network by:

1. allowing to switch off network elements (i.e. fibers, OXCs)

2. exploiting the cloud specific anycast principle.

Anycast is based on the principle that a cloud user is not concerned with the exact location of execution of her/his application, as long as the requirements of this application are met. Hence, we can optimize the selection of the IT-end point (and network resources to reach the destination) based on the associated power consumption by incorporating the minimization of energy in the routing objective.

In this paper, we evaluate the potential energy savings for an optical network with full wavelength conversion capability (WC) as well as for a network with a wavelength continuity constraint (i.e. without wavelength conversion, and assuming transparent switching; NoWC). Our main strategy to reduce power consumption is switching off unused resources, since in [3] it is shown that this can reduce the number of active links and nodes up to $25 \%$. The main contribution of our work is that we assess the additional impact on the energy budget by exploiting the anycast principle: we allow selecting traffic of a cloud user to be terminated at any of a given set of resources (as opposed to an a priori determined unicast traffic matrix, explicitly specifying both source and destinations). 


\section{2. ENERGY EFFICIENT ROUTING APPROACHES: MILP FORMULATION}

\begin{tabular}{|c|c|}
\hline$G=(N, V, L)$ & $\begin{array}{l}\text { Graph representing the optical network, with } N \text { the node } \\
\text { set, } L \text { the link set, } V \text { the resource site set. }\end{array}$ \\
\hline$P_{\text {conv }}$ & $\begin{array}{l}\text { Power consumption for an all-optical wavelength } \\
\text { converter }(=1.65 \mathrm{~W}) \text { (Note: this is set to } 0 \text { for NoWC) }\end{array}$ \\
\hline$P_{\text {edfa }}$ & Power consumption of an optical amplifier $(=9 \mathrm{~W})$ \\
\hline fibre span & $\begin{array}{l}\text { Length for which an optical signal needs to be } \\
\text { regenerated }(=80 \mathrm{~km})\end{array}$ \\
\hline$K$ & $\begin{array}{l}\text { The request set, indexed by } k \text { and } k_{n} \text { a request } \\
\text { originating at node } n \text {. }\end{array}$ \\
\hline$P_{\text {base }}$ & $\begin{array}{l}\text { Power for electronic control (e.g. GMPLS controller) }(= \\
150 \mathrm{~W})\end{array}$ \\
\hline$P_{\text {switch }}$ & $\begin{array}{l}\text { Energy consumption per wavelength for switching } \\
\text { devices (e.g. MEMS) [3] }=(107 \mathrm{~mW})\end{array}$ \\
\hline
\end{tabular}

Table 1 Parameters used in the MILP

The problem formulation can be stated as follows.

Given: the network topology with optical cross-connects (OXCs) and fiber links interconnecting them, including source sites (where requests for cloud services originate) and candidate destinations (i.e. the cloud servers),

Find: the routes for each of the requests such that power consumption is minimized.

Note that power consumption model described in [4] is used to calculate the total power consumption of optical network.

We will compare two routing approaches: (i) Unicast routing, where the destination of each request is fixed a priori, and (ii) Anycast routing, where we can freely choose any of the given cloud servers. We will further evaluate the anycast flexibility effect on energy usage, for two types of optical networks: (i) WC will refer to the case where we have wavelength converters in every OXC, and (ii) NoWC refers to a set-up where wavelengths are switched transparently and we need to respect wavelength continuity along the whole path. We model our routing scheme using Mixed Integer Linear Programming (MILP), for which the parameters and variables are described in Table 1. In what follows we describe the MILP for both the WC and NoWC case.

The objective function (1) minimizes the energy consumed by the network.

$$
\boldsymbol{m i n}\left(\sum_{n \in N} p_{n}+\sum_{l \in L} p_{l}\right)
$$

The constraints described in (2) enforce the link capacity constraints.

$$
w_{l}<W, \forall l \in L \text { (2) }
$$




\begin{tabular}{|c|c|}
\hline \multicolumn{2}{|r|}{ Wavelength conversion specific variables (WC) } \\
\hline $\begin{array}{l}w_{l}^{k} \in\{0,1\} \\
b_{n}^{k} \in\{0,1\}\end{array}$ & $\begin{array}{l}1 \text { if request } k \text { is routed over link } l, 0 \text { otherwise } \\
1 \text { if OXC } n \text { is the end node for connection } k, 0 \text { else }\end{array}$ \\
\hline \multicolumn{2}{|c|}{ Without wavelength conversion specific variables (NoWC) } \\
\hline$w_{l}^{\lambda, k} \in\{0,1\}$ & 1 if $k$ is routed over link $l$ using wavelength $\lambda, 0$ else \\
\hline$b_{n}^{\lambda, k} \in\{0,1\}$ & $\begin{array}{l}1 \text { if } \mathrm{OXC} n \text { is the end node for } k \text { and wavelength } \lambda \text { is } \\
\text { used, } 0 \text { else }\end{array}$ \\
\hline$c_{k}^{\lambda} \in\{0,1\}$ & 1 if wavelength $\lambda$ is used to route $\mathrm{k}, 0$ otherwise \\
\hline
\end{tabular}

Table 2: variables used by the MILP's

The next set of constraints is necessary to compute whether a link has active wavelengths (and consumes power).

$$
f_{l}<w_{l} \text { and } f_{l} \cdot W \geq w_{l}, \forall l \in L
$$

We do the same for the optical cross connects. We switch on an optical cross connect when one of its incident links is active. We denote $\omega(n)$ as the set of incident links of $n$ and $M$ the node degree of $n$.

$$
\begin{array}{ll}
y_{n} \leq \sum_{l \in \omega(n)} f_{l} & \forall n \in N \\
\sum_{l \in \omega(n)} f_{l} \leq M \cdot y_{n} & \forall n \in N(5)
\end{array}
$$

To compute the power of an optical cross connect, we add up the base power and the power needed to switch a signal.

$$
p_{n}=y_{n} \cdot P_{\text {base }}+\sum_{l \in \omega^{-}(n)} w_{l} \cdot\left(P_{\text {switch }}+P_{\text {conv }}\right), \forall n \in N
$$

For each link we compute the power as the power needed for the optical amplifiers.

$$
p_{l}=f_{l} \cdot P_{\text {edfa }} \cdot\left(2+\left\lceil\frac{|l|}{\text { fibre span }}\right\rceil\right) \quad, \forall l \in L \text { (7) }
$$

So far all the constraints are shared by both the WC and the NoWC MILP's. The next constraints, although stating the same concepts, have some minor difference between WC and NoWC.

We continue with the flow conservation constraints. We denote $S(n, k)=1 \Leftrightarrow n$ is $k$ 's source and $\omega^{+}(n)$ as the set of outgoing fibers and $\omega^{-}(n)$ the set of incoming fibers of OXC $n$ 


$$
\begin{gathered}
\sum_{l \in \omega^{+}(n)} w_{l}^{k}-\sum_{l \in \omega^{-}(n)} w_{l}^{k}= \\
\left\{\begin{array}{c}
-1 \text { if } S(n, k)=1 \\
b_{n}^{k} \text { else }
\end{array}\right.
\end{gathered}
$$

$\forall n \in N, k \in K(8)$

$$
\begin{aligned}
& \sum_{l \in \omega^{+}(n)} w_{l}^{\lambda, k}-\sum_{l \in \omega^{-}(n)} w_{l}^{\lambda, k}= \\
& \left\{\begin{aligned}
-c_{\lambda}^{k} \text { if } S(n, k)=1 \\
b_{n}^{\lambda, k} \text { else } \\
\forall n \in N, k \in K, \lambda<W(9)
\end{aligned}\right.
\end{aligned}
$$

Next we count the number of active wavelengths on a link.

WC

$w_{l}=\sum_{k \in K} w_{l}^{k} \quad \forall l \in L$
NoWC

(10) $w_{l}=\sum_{\lambda<W} \sum_{k \in K} w_{l}^{\lambda, k} \quad \forall l \in L$

Finally we end up with the resource demand constraints which ensure to choose only one resource node for each request (constraints 12,13) and to set the wavelength assignment for the specific destination in NoWC case (Constraints 16, 17). .

\section{WC}

$\sum_{n \in V} b_{n}^{k}=1 \quad \forall k \in K$

$$
b_{n}^{k}=\left\{\begin{array}{c}
1 \text { if } n \text { is } k^{\prime} s \text { destination } \\
0 \text { else } \\
\forall k K, \forall n \in N
\end{array}\right.
$$

$$
\sum_{\lambda<W} \sum_{n \in V} b_{n}^{\lambda, k}=1 \forall k \in K
$$$$
\sum_{\lambda<W} b_{n}^{\lambda, k}=\left\{\begin{array}{c}
1 \text { if } n \text { is } k^{\prime} \text { s destination } \\
0 \text { else } \\
\forall k \in K, \forall n \in N(15)
\end{array}\right.
$$

$$
\begin{aligned}
& \sum_{k \in K} w_{l}^{\lambda, k} \leq 1, \forall \lambda<W, \forall l \in L \\
& \sum_{k \in K} c_{\lambda}^{k} \leq 1,, \forall \lambda<W, \forall l \in L
\end{aligned}
$$

\section{USE CASES AND RESULTS}

We considered the Cost 239 European Network with 11 nodes and 26 bidirectional links with distances ranging from 270 to $1000 \mathrm{~km}$ and 3 server sites. Each fiber supports 16 wavelengths.

For the Unicast case, we generated 10 random demand matrices (for a total of 5 to 50 requests, where each request requires a full wavelength channel. We used these matrices also for anycast, but disregarded the specified destination. Power consumption parameters are set according to [3]. We plot the total energy consumptions for two cases, i.e. with wavelength conversion (WC) for both unicast and anycast cases in Fig. 1, and the corresponding number of wavelengths used in Fig. 2. We also computed the results for Shortest Path routing. 


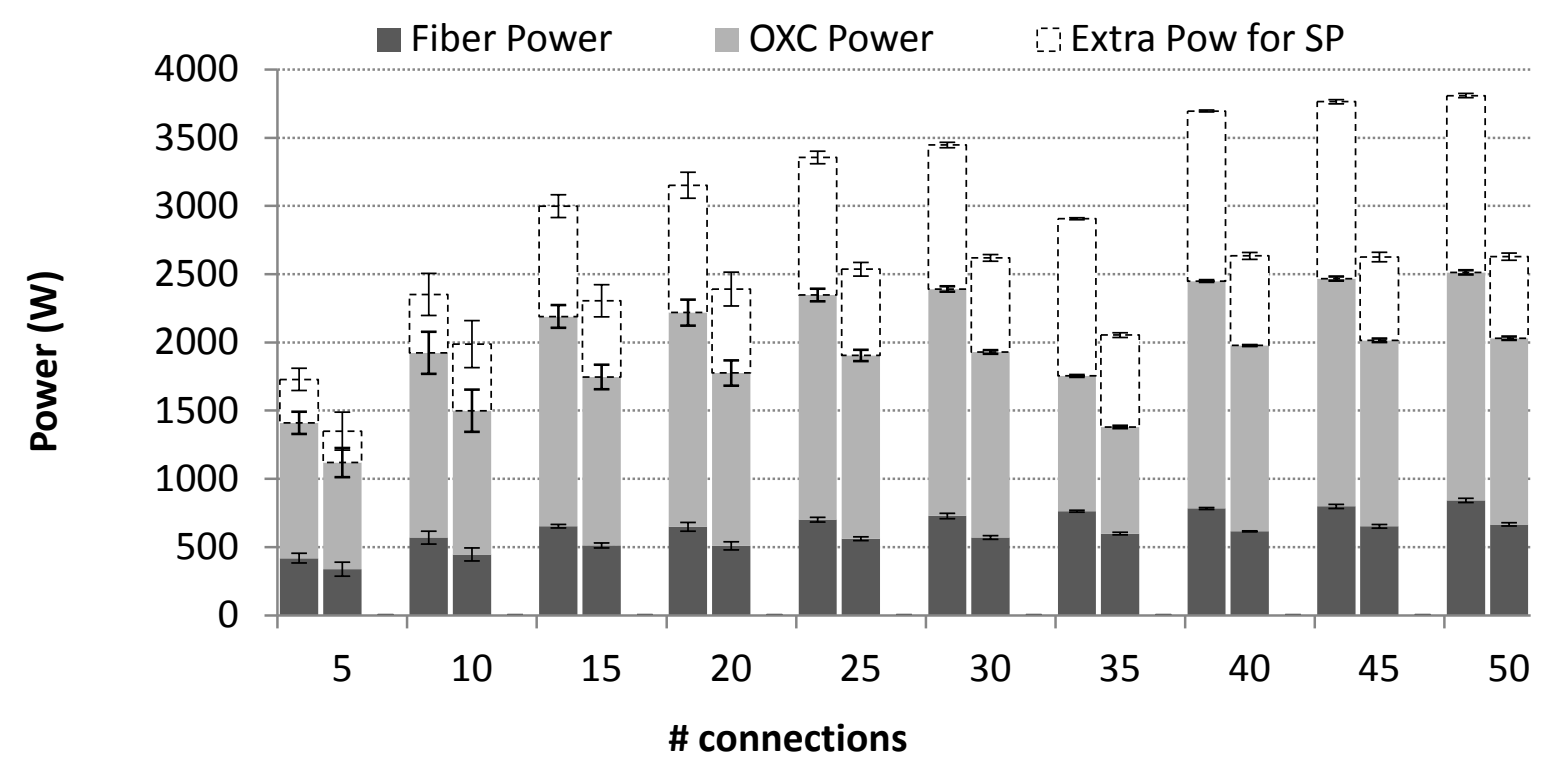

Fig. 1 - Power consumption for a WC network. Error bars indicate 95\% confidence intervals. The dotted lines represent the total energy cost when applying shortest path routing.

First, Fig. 1 illustrates the relative power consumed in nodes with respect to the fiber links: Power consumption in the fiber links accounts for 30\%, while OXC power constitutes about $70 \%$ of the total network energy consumption due to the traffic independent power consumption of the electronic control unit in the $\mathrm{OXC}, P_{\text {base }}$.

Secondly, energy-aware (EE) routing for both unicast and anycast approaches are compared with shortest path (SP) routing in Fig. 1. It is observed that EE routing helps to switch off more network resources and hence achieves better power savings. Dotted power bar depicts the extra energy needed for SP routing compared to EE routing. EE routing uses on average $23 \%$ less power for Anycast and 28\% less power for Unicast.

Thirdly in Fig. 1 we observe that by exploiting anycast, we can achieve an energy reduction of $20 \%$, both in fiber and node power, compared to the unicast case. Note that in this case, this benefit of around $20 \%$ is independent of the wavelength conversion capability (WC vs. NoWC) and load (i.e. number of connections). This benefit stems from the freedom in optimizing the destination site, thus achieving different (i.e. shorter) routings than the unicast case, which in turn permits to switch off more network elements (e.g. 100\% of OXCs are on when there are more than 25 connections in the unicast case, vs. around $80 \%$ for anycast). Such savings ( $29 \%$ ) also appear in wavelength resource usage (Fig. 2).

Finally, we remark that the relative difference between anycast and unicast remains similar for the NoWC case. Comparing them, we see that the power consumption is slightly higher in the WC case, mainly stemming from the wavelength converters. Yet, the advantage of NoWC is mitigated by the fact that due to its extra wavelength continuity constraints, NoWC cannot 


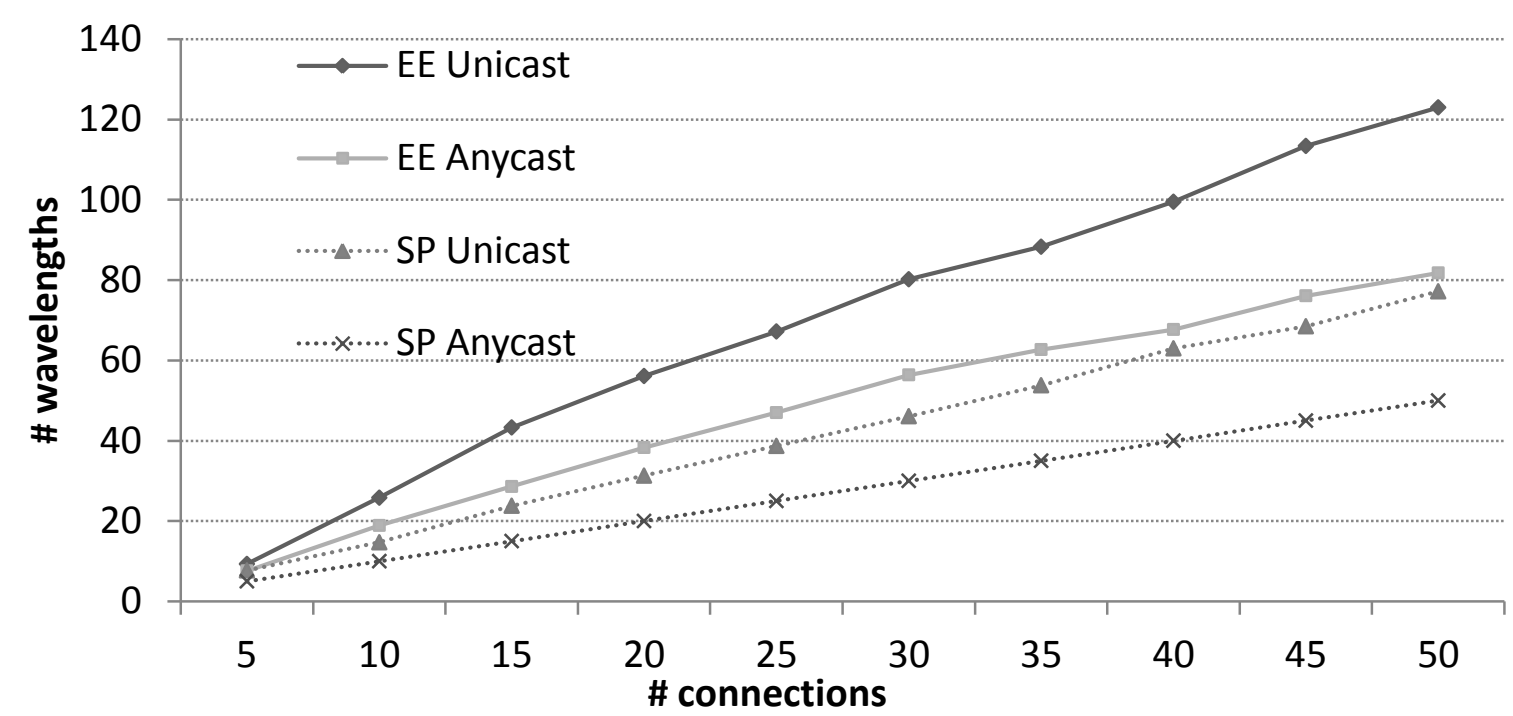

Fig. 2 - Total number of wavelengths, summed over all fibers, for a wavelength-conversion (WC) capable network.

always use the more energy efficient routes that are available in the WC case. We noted a net power reduction for NoWC up to around $170 \mathrm{~W}$ for 50 connections.

\section{CONCLUSIONS}

We propose an Energy Efficient routing approach for the case of optical cloud networks, which allows switching off idle network elements and exploits the anycast principle by allowing optimizing the destination site to route to. We have formulated our proposed EE routing solution using MILP and evaluated it on the European COST239 network, where we demonstrated that we can achieve on average $20 \%$ of energy reduction and $29 \%$ of wavelength resource usage compared to unicast routing, independent on the load or wavelength continuity constraints.

\section{REFERENCES}

1. M. Pickavet, et al., "Worldwide energy needs for ICT: the rise of power-aware networking", Proc. 2nd Int. Symp. Advanced Netw. and Telecommun. Systems (ANTS 2008), 15-17 Dec. 2008

2. L. Chiaraviglio, M. Mellia, F. Neri, "Energy-aware backbone networks: a case study", Proc. 1st Int. Workshop on Green Comm.. at IEEE Int. Conf. Commun. (ICC 2009), 18 Jun. 2009

3. S. Aleksić, "Analysis of power consumption in future high-capacity network nodes", J. Opt. Comm. Netw.

4. C. Cavdar, F. Buzluca L. Wosinska, "Energy-efficient design of survivable WDM networks with shared backup", Proc. IEEE Global Telecommun. Conf. (Globecom 2010), 6-10 Dec. 2010 . 